\title{
Valoración económica de los servicios ecosistémicos de recreación de la laguna de
}

\section{Chacas - Juliaca}

\section{Economic assessment of the ecosystemic recreation service of the lake of Chacas -}

\author{
Juliaca \\ José Eladio Núñez Quiroga ${ }^{1}$ y Karen Jenny Cutipa Quiso ${ }^{1}$
}

${ }^{1}$ Universidad Privada San Carlos. Jr. Lambayeque $\mathrm{N}^{\circ}$ 349, Puno, Perú. Correo electrónico: jenq1969@gmail.com_ORCID: https://orcid.org/0000-0002-7145-6625

\section{Resumen}

El objetivo fue estimar los beneficios económicos de los recursos eco sistémicos de la laguna ChacasJuliaca, se aplicó el método de valoración contingente basado en preferencias declaradas de los usuarios de la laguna, estos datos introducidos en un modelo econométrico logit, se valoró económicamente un servicio recreativo, determinándose una disposición a pagar. Los resultados muestran una tarifa de entrada de S/. 38 que los usuarios están dispuestos a pagar, esta disponibilidad depende variables sociales y económicas, como el ingreso y el grado de instrucción académico. Estos beneficios servirán para recuperar la laguna, permitiendo crear un fondo que permita conservar y proteger los recursos naturales de la laguna, actualmente existe una degradación biótica y abiótica por la contaminación de actividades como la pesca y la agricultura. Se requiere un financiamiento auto sostenible, para conservar este recurso natural, previniendo futuros deterioros del ecosistema de la laguna, permitiendo conservar sus recursos naturales.

Palabras clave: Laguna de Chacas - Juliaca, disponibilidad a pagar, teoría de las preferencias, servicios de recreación y valoración contingente.

\begin{abstract}
The objective was to estimate the economic benefits of the eco-systemic resources of the ChacasJuliaca lagoon, the contingent valuation method was applied based on the declared preferences of the users of the lagoon, these data were introduced in a logit econometric model, a recreational service, determining a willingness to pay. The results show an entrance fee of S /. 38 that users are willing to pay, this availability depends on social and economic variables, such as income and the degree of academic education. These benefits will serve to recover the lagoon, allowing to create a fund that allows to conserve and protect the natural resources of the lagoon, currently there is a biotic and abiotic degradation due to the contamination of activities such as fishing and agriculture. Selfsustaining financing is required to conserve this natural resource, preventing future deterioration of the lagoon's ecosystem, allowing it to conserve its natural resources.
\end{abstract}

Keywords: Laguna de Chacas - Juliaca, willingness to pay, preference theory, recreation services and contingent valuation. 


\section{Introducción}

En el planeta son muy importantes los ecosistemas en el desarrollo de nuestras sociedades mejorando el bienestar de las personas, estos pueden ser considerados como indicadores de la calidad o capacidad de un ecosistema para la provisión de un beneficio específico a un actor social determinado (Quétier, Tapella, Conti, Cáceres \& Díaz, 2007). De esta manera los servicios que tienen usos indirectos o valores intangibles han sido poco representados en las valoraciones monetarias (Briceño, Iñiguez \& Ravera, 2016).

Mantener los ecosistemas sin cambios con buen manejo permitirá una alta provisión de bienes y servicios ambientales, la producción económica inevitablemente afecta la provisión de servicios ecosistémicos (Farley, 2012). Es así que cualquier impacto negativo sobre el medio puede alterar el funcionamiento de los ecosistemas y reducir su capacidad de aporte local al entono y a la sociedad (Llerena \& Yalle, 2014).

Los recursos naturales como flora y fauna no cuentan con un estándar de precio en el mercado del Perú, por la inexistencia de un sistema mercantil donde puedan ser intercambiados, esto no quiere decir que carezcan de valor, por tanto, es necesario aplicar métodos de valoración que nos permita estimar dicho valor o contar con un indicador de su importancia en el bienestar de la sociedad, que permita compararlo con otros componentes del mismo, para lo cual será factible utilizar el dinero como denominador común (Raffo, 2015).

La Laguna Chacas es el habitad para un gran número de aves acuáticas y de aves estacionarias como el "flamenco de James" Phoenicoparrus jamesi, también alberga una cantidad de peces donde hay predominancia de la trucha arcoíris, según entrevistas a pescadores, existe la presencia de totora y totorilla, además de la presencia de ganado ovino, vacuno por la presencia de ganaderos en las comunidades aledañas, así mismo está rodeada por cerros cubiertos por pajonales y arboles como el eucalipto, todo lo anterior en conjunto genera un bienestar paisajístico y armonioso. 

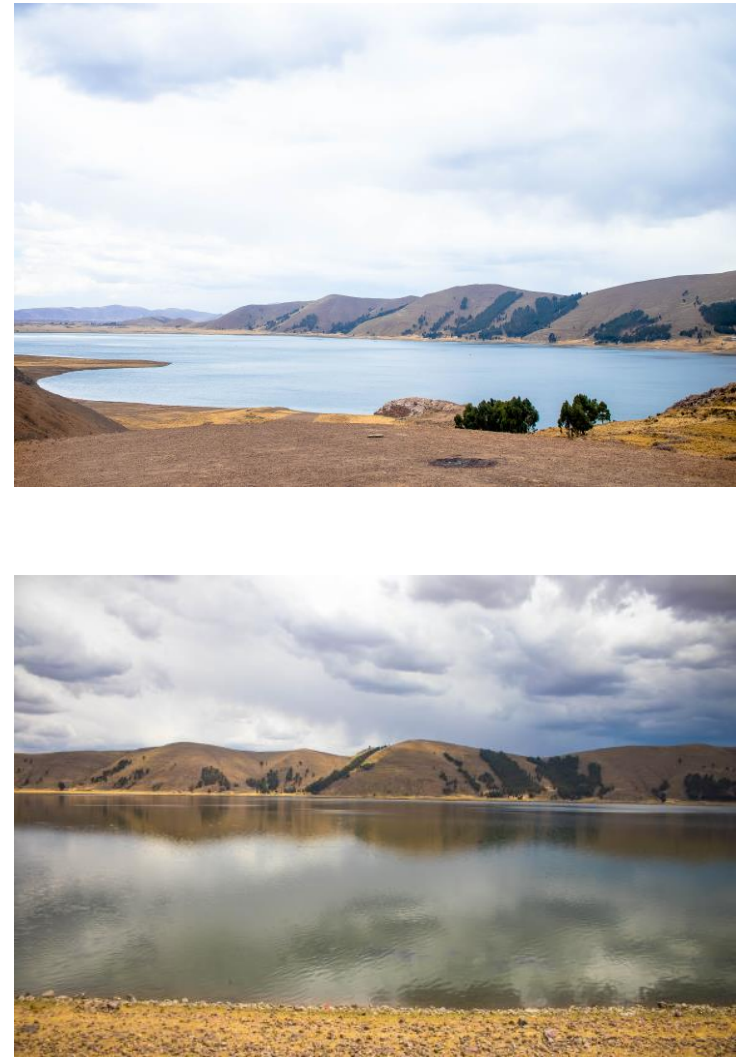

Figura 1: Vistas de la laguna Chacas

Fuente: Trabajo de campo

En general los servicios ecosistémicos hacen referencia al conjunto de beneficios que la sociedad obtiene de los ecosistemas, en forma de servicios en sentido estricto (regulación del clima, regulación de la composición atmosférica, formación de suelos, control de procesos hidrológicos, mantenimiento de recursos genéticos, etc.) o incluso de otros beneficios menos tangibles como los culturales, religiosos o espirituales, también bienes materiales (materias primas, alimento, energía fósil, etc.) (Daily, 1997; Millennium Ecosystem Assessment, 2005; Daniel et al., 2012).

La laguna de Chacas presenta un área de ecosistema ideal para servicios recreativos y ambientales, sin embargo, este ecosistema no tiene un precio de mercado, la valoración económica es un instrumento político útil para la toma de decisiones de planificación y gestión ambiental en general, porque lleva la discusión al terreno monetario, pero casi siempre infra o subvalora el ambiente (Iwan, Guerrero, Romanelli, \& Bocanegra, 2017). Como no toda la superficie de la laguna es área protegida provincial, en la cuenca coexisten otros usos entre los que se destacan: asentamientos urbanos, explotaciones agrícola-ganaderas extensivas e intensivas como la frutihortícola- y espacios de conservación (Bó et al. 2009; Romanelli, Lima. Londoño, Martinez \& Masso, 2012).

El marco teórico se sustenta en una función de bienestar social que convierte los niveles de utilidad para todos los miembros de la sociedad en un número de tal forma que la función de bienestar asigna mayores valores a las distribuciones de utilidad que son socialmente más convenientes y viceversa, en una economía de trueque de bienes es la 
misma para todos los participantes, lo que supone que el consumo de un bien aumenta en una pequeña cantidad y el del otro bien disminuye, de tal manera que la utilidad permanece constante y la proporción de estos dos cambios es la tasa marginal de sustitución. Cuando los precios se utilizan para intercambio en una economía de dos bienes, las cantidades para el equilibrio de los bienes generalmente será el punto de la curva contrato en el cual la línea presupuestal es igual a la tasa marginal sustitución para cada consumidor, y donde la línea presupuestal pasa a través de la dotación inicial. El excedente total de incrementar la cantidad ofrecida de $\mathrm{q}^{*}$ a $\mathrm{q}^{* *}$ es el área limitada arriba por la curva de demanda (Kolstad, 2001).

A veces es complicado asignar un valor real a bienes y servicios que no tienen mercado (López, 2015), el término surge de la idea de compensar los daños ambientales y sociales a través de herramientas de gestión ambiental apropiadas, por eso la valoración económica casi siempre infra o subvalora el ambiente; no obstante, puede ser un instrumento político útil para la toma de decisiones de planificación y gestión ambiental en general porque lleva la discusión al terreno monetario (Iwan et al., 2017), para (Sarmiento, 2004), en las últimas décadas los economistas han notado la falta de asignarle un precio a los medios naturales y beneficios ambientales para contribuir información propenso a corregir algunos de los graves problemas ambientales que aquejan al naturaleza.

Existen dos enfoques para obtener información sobre la demanda y por tanto el valor de los bienes y servicios ambientales estos enfoques son los métodos directos y los métodos indirectos (Castiblanco, 2003), el método de Valoración Contingente, es utilizado para valorar los beneficios de una mejora ambiental de acuerdo con la cantidad monetaria que los beneficiarios potenciales de dicha mejora estarían dispuestos a pagar (Abson \& Termansen, 2011), el modelo logit multinomial descansa sobre un supuesto muy restrictivo: la independencia entre alternativas irrelevantes. Es decir, la probabilidad de una determinada alternativa no se ve afectada por la existencia de alternativas adicionales (Rierafont, 2008).

Tansley (1935) acuño el término ecosistema como el complejo de organismos junto con los factores físicos de su medio ambiente en un lugar determinado, y planteado también como una de las unidades básica de la naturaleza, a partir su diseño resaltó la idea de ecosistema como "unidad básica de la naturaleza". 
Armenteras et al. (2015) sostiene que a partir de su concepción inicial, el ecosistema ha sido ampliamente utilizado como marco de referencia para entender cómo funcionan los seres vivos y su medio ambiente, hasta llegar a ser propuesto como concepto de organización, marco y teoría central en la ecología o como una estrategia para la gestión de los recursos, su conservación y uso de manera equitativa.

Sepúlveda et al. (2010), el turismo puede ser estudiado desde diversas perspectivas y disciplinas, debido a que son muchos los elementos que lo constituyen. A partir que el turismo comienza a atraer como materia de estudio profesional, existe una discusión directa para querer alcanzar un concepto consensuado del vocablo Turismo y que éste quede plasmado como una enunciación mundial.

Los servicios ecosistémicos son definidos como los beneficios ambientales, sociales, económicos, directos e indirectos, que las personas obtienen del buen funcionamiento de los ecosistemas. Entre ellos se cuenta la regulación hídrica en cuencas, el mantenimiento de la biodiversidad, el secuestro de carbono, la belleza paisajística, la formación de suelos y la provisión de recursos genéticos, entre otros (Ley $\mathrm{N}^{\circ}$ 30215, Ley de Mecanismos de Retribución por Servicios Ecosistémicos).

Firozan, Bahamanpour, Hakini y Hashimi (2012), utilizaron el método de valoración contingente para evaluar el valor recreativo de Sari Forest Park (Irán) en 2012, teniendo en cuenta las numerosas funciones y servicios prestados por los bosques y parques forestales. Para ello, la DAP de los visitantes se estimó utilizando el método de valoración contingente exhaustivo a través de un cuestionario dicotómico. Para el cálculo de la DAP el modelo utilizado fue Logit, el resultado mostró que la media DAP de los visitantes para el valor recreativo del parque fue 8,216 reales por visitante y su valor total anual fue de 123 mil millones de reales. El resultado también revelo que los parámetros de oferta y los ingresos fueron significativos desde un punto de vista estadístico, las cuales fueron los factores más eficaces en la DAP de los visitantes. Además, con el aumento de las atracciones forestales, la DAP creció en $47 \%$, donde los resultados indicaron un interés de los visitantes en las áreas recreativas y parques forestales, que se puede tomar en consideración cuando se gestionan estos ecosistemas. 
Sarmiento, Rodríguez y Rivera (2015), estimó

la DAP de los visitantes de la Laguna Sausachoca con la metodología de valoración contingente asumiendo que esta sería para conservar la calidad ambiental del recurso, es así que en los resultados se manifestó que el $59 \%$ de encuestado reconocen que los servicios ecosistémicos son algo importantes, mientras que un $41 \%$ manifestó que son bastante importantes. El valor promedio del pago está en S/. 7,60 y se encontró un valor máximo de S/. 50. La desviación estándar del valor medio está en S/. 7, 96.

Verona y Rodríguez (2013), identificó los bienes y servicios ambientales, encontrando un valor económico aproximado con el valor de disfrute de las personas que visitan la Laguna Conache, empleando el método de valoración contingente, se estimó que el valor anual declarada de DAP por conservar la Laguna fue de S/. 2,808 937,66 nuevos soles; y el valor anual que las personas creen que vale su disfrute fue de S/. 4,185 720,00 nuevos soles. La diferencia se debe a que a las personas se les está pidiendo que paguen por algo y a nadie le encanta la idea de gastar, pero en cambio cuando se refieren al valor de su disfrute valoración es mayor por lo que no se les está hablando de pago alguno.
Aguilar (2015), estimó la DAP con el uso de la metodología de valoración contingente, a través de la aplicación de 107 encuestas a visitantes de la Laguna de Pacucha, se determinó con la probabilidad del 9,8\%, que los visitantes están dispuestos a pagar S/. 1,00 nuevo sol por cada visita esto para la conservación, y mejora de sus servicios turísticos que se brindan.

Vera (2013), estimó la disposición a pagar por el usufructo en dos estaciones (verano e invierno) para la Laguna Punta Porã donde la DAP media para invierno es de 99889 guaraníes por mes, el valor de uso anual fue de aproximadamente de 1,388,057,11. La DAP media para la estación de verano es de 150889 de guaraníes por mes, y el valor de uso anual fue de aproximadamente de 2,096,753,55 guaraníes. Los datos obtenidos servirán para la toma de decisiones estratégicas a nivel del sitio y por parte de las autoridades que tengan competencia en su manejo.

Poner en vitrina los servicios recreativos del ecosistema de la laguna de Chacas, para la población de Juliaca, estas actividades recreativas naturales permitirán generar ingresos que deducidos los costos podría generar utilidades para actividades de turismo en el sector privado y público, entonces 
necesitamos conocer la disponibilidad a pagar del poblador de Juliaca, para estimar los beneficios producto de actividades de servicios naturales que brindan la laguna de Chacas. El objetivo general de esta investigación es dimensionar estimar los beneficios sociales provocados por la adecuación de un servicio turístico que impactaría los atributos de la laguna de Chacas.

\section{Metodología}

La Laguna de Chacas ubicada en la región Puno, provincia de San Román, distrito de Juliaca en el área concerniente a los poblados de Kokan y Chacas, pertenece a la cuenca hidrográfica del Río Coata. Ubicada a 10 km al noroeste de la ciudad de Juliaca (-15,410815 - 70,213801), el clima de la zona es frio, pero exquisito; una brisa agradable nace de la laguna; los ríos que desembocan en ella son pequeños y temporales.

La población a la que está dirigida la encuesta es la ciudad de Juliaca, según datos de INEI la población total comprendida en estas edades es de 177,324. Para la determinación del tamaño de muestra se utiliza la fórmula de proporciones, por lo tanto, se realizaron 383 encuestas, se sugiere que esté en los límites de edad estén entre los 18 - 65 años de edad, puesto que estas personas tendrían la disponibilidad a visitar la laguna de manera recreacional.

Se hizo un reconocimiento de los servicios ecosistémicos de la Laguna de Chacas y se utilizó el diagnóstico socio cultural de la microcuenca de Chacas de Suma Marka ONGD para reforzar la identificación de actividades antrópicas entorno a esta laguna, estos facilito los análisis de una correcta identificación y determinación de los servicios ecosistémicos.

Se utilizó el método de valoración contingente puesto que la orientación metodológica de esta investigación es analítica explicativa, con herramientas cualitativas y cuantitativas, en donde se indaga la disponibilidad a pagar (Tequia \& Camargo, 2016) en el cual el ciudadano usuario del servicio se enfrenta a un modelo hipotético expresando su disposición a pagar como un indicador de cambios en el bienestar frente al servicio (Agüero et al. 2008)

La valoración económica contempla los bienes o servicios generados por las áreas naturales, para esto es necesario internalizar las externalidades positivas o negativas de su utilización, (Garzón, 2013). Da un enfoque más sustantivo es conocer de qué manera la 
disponibilidad a pagar varía por factores que se esperaría tuvieran una influencia razonable en ésta. Ello resaltará en conexión al efecto incrustación (Embending effect) y la elasticidad ingreso de la disposición a pagar (Haneman, 2016).

Se construyó un mercado hipoteco para utilizar el método de valoración contingente, donde el consumidor es el demandante y el entrevistador es el ofertante, el cuestionario puede tener diferentes formatos para realizar la pregunta de la disponibilidad a pagar del consumidor. Primero se utilizó el formato abierto haciendo una pregunta abierta sobre la disponibilidad a pagar, estas respuestas sirvieron para tener una media aproximada de la disponibilidad a pagar del consumidor, en seguida se utilizó el formato referéndum que se deja al consumidor solamente con la alternativa de decidir si está dispuesto a pagar o no una suma determinada por arribar al servicio ambiental que se ofrece.

El problema de este método radica en que la respuesta del consumidor tiene que ser objetiva relacionado con el incentivo o desincentivo a revelar el "verdadero" precio, lo que ha motivado algunas críticas al método (Riera, 1994). Al resolver la pregunta de cuanto está dispuesto a pagar por este servicio, tal como si estuviera en un mercado, pero sin realizar el gasto en efectivo, estamos construyendo un mercado hipotético.

\section{Resultados y discusión}

La encuesta contiene tres cuerpos, el primero información social del consumidor como edad, sexo, lugar de residencia, número de personas que viven en su hogar, grado de instrucción, ocupación e ingreso; el segundo cuerpo conocimiento de la laguna de Chacas, de no tener idea del recurso se le alcanza la información mediante un brochure con imágenes de la carretera, de la laguna, tipos de aves, vegetales, aparcamiento o camping y otras características del ecosistema; finalmente el tercer cuerpo la posibilidad de vivir la experiencia de visitar la laguna de Chacas y la disponibilidad a pagar bajo formato de referéndum.

Mediante la prueba estadística el grado de significancia entre las variables el estadístico LR es 147.05 y con 6 grados de libertad el valor crítico de la chi-cuadrado al 5\% de significancia es 12,5916, el chi-cuadrado es mayor que el calculado por lo que se rechaza la hipótesis conjunta de que los coeficientes de todas las variables explicativas sean cero. 


\section{Tabla 1}

Resumen resultados modelos logit binomial

\begin{tabular}{lccc}
\hline \multicolumn{1}{c}{ Variable } & Coefficient & Standard Error & $\mathbf{P}[\mathbf{Z} \mid>\mathbf{z}]$ \\
\hline Constante & $-0,651$ & 0,215 & 0,7627 \\
Probabilidad de responder SI & $-0,022$ & 0,024 & 0,0000 \\
Ingreso & 0,123 & 0,034 & $0,0004^{*}$ \\
Grado de instrucción & 0,163 & 0,050 & $0,0012^{*}$ \\
Sexo & 0,029 & 0,067 & 0,6634 \\
Edad & $-0,007$ & 0,025 & 0,7697 \\
Personas que viven en el hogar & 0,016 & 0,0150 & 0,2689 \\
\hline
\end{tabular}

Los números $\mathrm{P}[|\mathrm{Z}|>\mathrm{Z}]$ son los t-estadístico. * Significancia al 1\%

Teniendo en cuenta los resultados mostrados

en la tabla 1 , los signos de los coeficientes son los esperados, la probabilidad de responder SI es negativo lo que indica que a mayor precio la respuesta del consumidor es negativa, el signo del coeficiente de la variable ingreso (ING) es positivo lo que significa que si el encuestado tiene un mayor ingreso está dispuesto a realizar el servicio recreativo propuesto, la variable instrucción (INSTRUC) también tiene signo positivo entonces a mayor especialización el individuo valora este tipo de actividad, son los hombres que viven en la ciudad de Juliaca los que se inclinan más que las mujeres por este servicio recreativo, mientras que los más jóvenes están más dispuestos a pagar por visitar la laguna de Chacas, si bien es cierto la variable número de personas que viven en el hogar $(\mathrm{PVH})$ resulto positiva, podríamos señalar que las familias de tamaño grande tienen infantes lo que hace propicio este servicio recreativo para esas edades.

Es necesario señalar que las variables disponibilidad a pagar e ingreso son significativas al $1 \%$, mientras que la variable instrucción es significativa al 10\%.

El modelo predice correctamente en un 77,23 según el porcentaje de predicción de los resultados del modelo económetrico y el Pseudo R cuadrado con un $31,03 \%$ nos muestra un buen ajuste del modelo a la variable que se quiere explicar.

Los efectos marginales para las variables analizadas en el estudio de investigación, considerando aquellas variables significativas, podemos señalar que si hay un aumento en precio de la actividad recreativa, disminuye la probabilidad de aceptar este valor en un 2,20\% por parte del consumidor, por otro lado si mejora el ingreso del encuestado existe la 
probabilidad de responder positivamente en un $12,31 \%$ mayor y según el último grado de instrucción aprobado (INSTRUC) una mayor especialización y conocimientos del usuario permite elevar la respuesta afirmativa en un $16,37 \%$.

Tabla 2

Efectos marginales para las variables observadas

\begin{tabular}{lc}
\hline \multicolumn{1}{c}{ Variables } & Coeficientes \\
\hline Constante & $-0,06516$ \\
Probabilidad de responder & $-0,02203$ \\
si & \\
Ingreso & 0,12312 \\
Instrucción & 0,16370 \\
Sexo & 0,02926 \\
Edad & $-0,0754$ \\
Personas que viven en el & 0,01665 \\
hogar & \\
\hline
\end{tabular}

Una vez analizado y dado la conformidad del modelo econométrico, podemos encontrar la disponibilidad a pagar del consumidor de la ciudad de Juliaca por visitar y percibir mediante un servicio recreativo el ecosistema de la laguna de Chacas.

De acuerdo a los resultados, la disponibilidad a pagar por el usuario para este servicio recreativo en la laguna de Chacas es de S/. 38 con una disposición mínima a pagar de S/. 12 y una disposición máxima a pagar de S/. 61.

\section{Conclusiones}

Los beneficios no materiales que el consumidor de la ciudad de Juliaca obtiene de los ecosistemas de la laguna de Chacas, tales como la belleza escénica, la información para el desarrollo del conocimiento, la recreación y el turismo, está en función de variables como: el ingreso, el grado de instrucción académico, el sexo, la edad y el número de personas que viven en el hogar. Esta utilidad del consumidor se ve reflejada en la disponibilidad a pagar por una tarifa de entrada por un servicio recreativo en la laguna de Chacas, por un monto de S/. 38 , con un intervalo de confianza de S/. 12 a 61 , donde las variables el ingreso, el grado de instrucción académico son las que más influyen en el modelo econométrico, ahora teniendo en cuenta que en promedio los operadores turísticos privados de la región de Puno, tienen como media el cobro de un paquete similar en S/. 30, podemos concluir que el rendimiento para operadores privados y públicos sería alto, justamente para este último sector permitiría crear un fondo que permita conservar y proteger los recursos naturales de la laguna, además sustituir la actividad turística por otras actividades de degradación 
de los organismos vivos que interactúan entre

sí y con su entorno físico como una unidad geológica de la laguna.

\section{Referencia bibliográfica}

Abson, D., \& Termansen, M. (2011). Valuing Ecosystem Services in terms of ecological risk and returns. Conservation Biology, 25(2), 250258.

Aguilar, R. (2015). Cálculo de la disposición a pagar por la conservación y mejora de los servicios turísticos de la Laguna Pacucha. Ciencia y Desarrollo, 20, 7176.

Armenteras, D., Gonzáles, T., Vergara, L., Luque, F., Rodríguez, N., \& Bonilla, M. (2015). Revisión del concepto de ecosistema como "unidad de la naturaleza" 80 años después de su formulación. Ecosistemas, 25(1), 8389. doi:10,7818/EOS2016.25-1.12

Bó, M., del Rio, J.L., López de Armentia, A., Cionchi, M., Osterrieth, M., Álvarez, M. y Camino, M. (2009). Aplicación de un sistema de geoindicadores para la evaluación de la calidad ambiental en agroecosistemas. Revista de geología Aplicada a la Ingeniería (23) 55-66. http://www.editoresasagai.org.ar/sites /default/files/Revista-ASAGAI-

23_Mayo-2009_0.pdf

Briceño, J., Iñiguez, V., \& Ravera, F. (2016).

Factores que influyen en la percepción de servicios de los ecosistemas de los bosques secos del sur del Ecuador. Ecosistemas, 25(2), 46-58. doi:10.7818/ECOS.2016.25-2.06

Castiblanco, C. (2003). Los métodos de valoración económica del medio ambiente: conceptos preliminares. Ensayos de Economía, 13(1), 9-41.

Daniel, T., Muhar, A., Arnberger, A., Aznar, O., Boyd, J. Chan, K. y GrêtRegamey, A. (2012). Contributions of cultural services to the ecosystem services agenda. Proceedings of the National Academy of Sciences, 109(23), 8812-8819. https://doi.org/10.1073/pnas.1114773 109

Daily, G. (1997). Nature's services: societal dependence on natural ecosystems. Washington: Island Press.

Firozan, A., Bahamanpour, H., Hakimi, A., \& Hashimi, S. (2012). Estimated recreational value of Lahijan forest using by contingent valuation method. 
ARPN Journal of Agricultural and Biological Science, 7(9), 659-663.

Garzón, L. P. (2013). Revisión del Método de valoración contingente experiencias de la aplicación en áreas protegidas de América Latina y el Caribe. Espacio y Desarrollo, 25, 65-78.

Haneman, M. (2016). Valuando el medio ambiente a través de la valoración contingente. Gaceta Económica, 2, 17-53.

Iwan, A., Guerrero, M., Romanelli, A., \& Bocanegra, E. (2017). Valoración económica de los servicios ecosistémicos de una laguna del sudeste bonarense (Argentina). Investigaciones geográficas, 68, 173189. doi:10.14198/INGEO2017.68.10

Kolstad, Ch. (2001). Economía Ambiental. México D.F. Mexicana.

Ley $\mathrm{N}^{\circ}$ 30215, Ley de Mecanismos de Retribución por Servicios Ecosistémicos. Perú, Capítulo 1, Artículo 3 (2014).

Llerena, C., \& Yalle, S. (2014). Los servicios ecosistémicos en el Perú. Xilema, 27, 62-75.

López, G. (2015). Valoración económica del Servicio Ambiental de captura de Carbono en el Fundo Violeta (Tesis de grado). Pontificia Universidad Católica del Perú. Lima, Perú.

Millennium Ecosystem Assessment [MEA] (2005). Ecosystems and Human WellBeing: Synthesis (Island Press, Washington, DC).

Quétier, F., Tapella, E., Conti, G., Cáceres, D., \& Díaz, S. (2007). Servicios ecosistémicos y actores sociales, aspectos conceptuales y metodológicos para un estudio interdisciplinario. Gaceta Ecológica (84-85), 17-26.

Raffo, E. (2015). Valoración económica ambiental: El problema del costo social. Industrial data, 18(1), 108-118.

Riera, P. (1994). Manual de Valoración Contingente. Instituto de Estudios Fiscales.

Rierafont, A. (2008). Valoración económica de los atributos ambientales mediante el método del coste de viaje. Estudios de Economía aplicada, 14, 173-198.

Romanelli, A., Lima, M. L., Londoño, O. M. Q., Martínez, D. E., y Massone, H. E. (2012). A GIS-based assessment of groundwater suitability for irrigation purposes in flat areas of the Wet Pampa Plain, Argentina. Environmental Management, 50(3), 
490-503.

https://doi.org/10.1007/s00267-012-

9891-9

Sarmiento, M. (2004). Valoración económica ambiental de servicios recreativos del Lago Termas de Rio Hondo, Santiago del Estéreo. Método de valoración contingente versus costo de viaje. Asociación Argentino Uruguaya de Economía ecológica, 2, 1-16. doi:10.13140/2.1.1715.2001

Sarmiento, M., Rodríguez, A., \& Rivera, S. (2015). Valoración económica y ambiental de servicios ecosistémicos generados por la Laguna Sausachoca, Perú, Una aproximación mediante el método de valoración contingente. Estudios ambientales, 3(1), 16-37.

Sepúlveda, H., Basurto, R., \& Vizcarra, Y. (Junio de 2010). Plan estratégico para el desarrollo del turismo rural comunitario en la región Cusco (Tesis de maestría). Pontificia Universidad Católica del Perú. Surco, Lima, Perú. en Soacha, Cundinamarca, Colombia. Investigación agraria y ambiental, $7(2)$,

$171-183$

doi:10.22490/21456453.1566

Vera, C. (2013). Beneficio económico de la Laguna Punta Porã (Tesis Maestría). Universidad Nacional Asunción Filial Pedro Juan Caballero. Paraguay.

Verona, A., \& Rodríguez, C. (Enero de 2013). Valoración económica de bienes y servicios ambientales de la Laguna Conache, Laredo- La Libertad, Perú. Rebiolest, 1(1), 54-70. 